Research Paper

\title{
Gender Differences in Histamine-Induced Depolarization and Inward Currents in Vagal Ganglion Neurons in Rats
}

Jun-Nan Li ${ }^{1}$, Zhao Qian ${ }^{1}$, Wen-Xiao Xu ${ }^{2}$, Bing Xu${ }^{1}$, Xiao-Long Lu ${ }^{1}$, Zhen-Yu Yan ${ }^{1}$, Li-Min Han ${ }^{1}$, Yang Liu ${ }^{1,3}$, Mei Yuan ${ }^{3}$, John Schild ${ }^{4}$, Guo-Fen Qiao ${ }^{1,3}{ }^{凶}$, Bai-Yan Li1 $^{1}{ }^{凶}$

1. Department of Pharmacology, Harbin Medical University, Harbin, China,

2. Dept. of Orthopedics, the First Affiliated Hospital, Harbin Medical University, Harbin, China,

3. Key Laboratory of Cardiovascular Medicine Research, Ministry of Education, Harbin Medical University, Harbin, China

4. Department of Biomedical Engineering, Indiana University Purdue University Indianapolis, Indianapolis, USA

$\triangle$ Corresponding author: G-F Qiao (qiaogf88@163.com) or B-Y Li (baili@iupui.edu), Department of Pharmacology, Harbin Medical University, 157 Bao-Jian Road, Harbin 150081, China; Phone: 86-451-8667-1354; Fax: 86-451-8667-1234.

() Ivyspring International Publisher. This is an open-access article distributed under the terms of the Creative Commons License (http://creativecommons.org/ licenses/by-nc-nd/3.0/). Reproduction is permitted for personal, noncommercial use, provided that the article is in whole, unmodified, and properly cited.

Received: 2013.09.04; Accepted: 2013.11.06; Published: 2013.11.20

\begin{abstract}
Evidence has shown gender differences regarding the critical roles of histamine in the prevalence of asthma, anaphylaxis, and angina pectoris. Histamine depolarizes unmyelinated C-type neurons without any effects on myelinated A-type vagal ganglion neurons (VGNs) in male rats. However, little is known if VGNs from females react to histamine in a similar manner. Membrane depolarization and inward currents were tested in VGNs isolated from adult rats using a whole-cell patch technique. Results from males were consistent with the literature. Surprisingly, histamine-induced depolarization and inward currents were observed in both unmyelinated C-type and myelinated Aand Ah-type VGNs from female rats. In Ah-type neurons, responses to I.0 $\mu \mathrm{M}$ histamine were stronger in intact females than in males and significantly reduced in ovariectomized (OVX) females. In C-type neurons, histamine-induced events were significantly smaller $(\mathrm{PA} / \mathrm{pF})$ in intact females compared with males and this histamine-induced activity was dramatically increased by OVX. Female A-types responded to histamine, which was further increased following ovariectomy. Histamine at $300 \mathrm{nM}$ depolarized Ah-types in females, but not Ah-types in OVX females. In contrast, the sensitivity of A- and C-types to histamine was upregulated by OVX. These data demonstrate gender differences in VGN chemosensitivity to histamine for the first time. Myelinated Ah-types showed the highest sensitivity to histamine across female populations, which was changed by OVX. These novel findings improve the understanding of gender differences in the prevalence of asthma, anaphylaxis, and pain. Changes in sensitivity to histamine by OVX may explain alterations in the prevalence of certain pathophysiological conditions when women reach a postmenopausal age.
\end{abstract}

Key words: chemosensitivity, histamine, vagal afferent neuron, membrane depolarization, inward current, whole-cell patch.

\section{Introduction}

Histamine is an organic nitrogen compound involved in local immune responses (1) that can regulate physiological functions in the gut (2) and act as neurotransmitters to play a functional role in the neurocontrol of circulation (3-6). Histamine is also involved in many pathophysiological situations, such as vasospastic or pre-infarction angina $(7,8)$, neuropathic pain or inflammation (9-12), ischemic heart 
disease $(13,14)$, hypertension $(15,16)$, and allergic reactions $(17,18)$. Increasing evidence has shown significant gender differences in the incidence and clinical management of angina and asthma (19-23). However, underlying mechanisms at the cellular and molecular levels in the visceral afferent nerve system are not yet understood because most of the published data have been collected from male animals or animals with unspecified gender. Early studies demonstrated that nearly $25 \%$ of unmyelinated C-type nodose neurons from male cats respond to histamine, but that myelinated A-type neurons do not (24). Similarly, recent observations have shown that vagal nodose esophageal C-fibers from male guinea pigs can be excited by histamine (25) and that more than $20 \%$ of neurons within the nucleus of the tractus solitaries (NTS) in male rats were depolarized by histamine (26). Controversial responses to histamine have previously been reported in cardiac afferent neurons in the nodose ganglion of guinea pigs of unknown sex (27). Consistently, histamine receptors (H1) have been identified in both the nodose ganglion (28) and NTS (6). The reason why scientists have not been able to fully understand the possible gender differences may be due in large part to the predominant use of male animals. The present study explored gender differences in histamine-induced activity in myelinated and unmyelinated vagal ganglion neurons (VGNs) from adult age-matched male and female (with ovaries intact and surgically removed) rats under currentand voltage-clamp modes using a whole-cell patch technique. Because compelling evidence in the literature has shown that the prevalence of asthma is significantly higher in boys than girls and that this pattern reverses in adulthood $(29,30)$, VGNs isolated from neonatal rats were also evaluated with histamine to better understand the underlying mechanisms of these clinical observations. The novel findings from the present study provide the first direct electrophysiological and pharmacological evidence demonstrating gender differences in chemosensitivity of neurons to histamine, which may partially explain gender differences in autonomic reflex function or dysfunction and the incidence of clinical disorders, such as angina, asthma, allergies, and neuropathic pain.

\section{MATERIALS and METHODS}

\section{Animals}

All experimental animals used in this study, including adult male and intact and ovariectomized (OVX) female Sprague Dawley (SD) rats, were obtained from the animal facility at Harbin Medical University in China or purchased from Harlan La- boratories (Indianapolis, IN, USA). Experimental protocols were pre-approved by the Institutional Animal Care and Use Committee of School of Medical Science at Harbin Medical University and Indiana University.

\section{Drug and chemical agents}

Histamine was purchased from Tocris (Ellisville, $\mathrm{MO}, \mathrm{USA})$. Histamine stock solution at $100 \mu \mathrm{M}$ was prepared using recording solution and stored at $4^{\circ} \mathrm{C}$. Histamine was applied through bath perfusion $(\sim 1.0$ $\mathrm{ml} / \mathrm{min}$ ). Papain (Sigma, St. Louis, MO), type II collagenase (Worthington Biochemical, Lakewood, NJ), and dispase (Roche, Mannheim, Germany) were used for enzymatic isolation of vagal neurons from rats. Mg-ATP and Na-GTP (Sigma, St. Louis, MO) were used to prepare the pipette solution. Earle's balanced salt solution (Sigma) was selected to dissolve enzymes. All other cell culture reagents and chemicals were obtained from Fisher Scientific or other sources as specified.

\section{Preparation of isolated vagal ganglion neurons from adult rats}

All procedures followed the protocols described previously (31). Briefly, after both sides of the nodose ganglia were surgically removed, they were immediately placed into chilled $\left(\sim 4^{\circ} \mathrm{C}\right)$ recording solution. Connective tissue around the ganglia was carefully removed and each ganglion was cut into small pieces under a surgical microscope (x40). Ganglion tissue was transferred to an enzyme solution containing 20 units $/ \mathrm{ml}$ of papain in a $15 \mathrm{ml}$ centrifuge tube and digested at $37^{\circ} \mathrm{C}$ in a moisturized environment with $95 \% \mathrm{CO}_{2}$ and $5 \% \mathrm{O}_{2}$ for $20 \mathrm{~min}$. The ganglion tissue was transferred to another enzyme solution containing $1.0 \mathrm{mg} / \mathrm{ml}$ type II collagenase and $2.0 \mathrm{mg} / \mathrm{ml}$ dispase and incubated under the same conditions for additional $30 \mathrm{~min}$. After digestion, the enzyme solution was completely removed and $1.0 \mathrm{ml}$ of warm $\left(\sim 37^{\circ} \mathrm{C}\right)$ nodose complete medium $(\mathrm{NCM})$ was added and gently triturated with a glass aspiration pipette until the ganglion tissue disappeared. The cell suspension was then placed onto a poly-D-lysine coated cover slip (10x10) and incubated for at least $60 \mathrm{~min}$ for patching. NCM contained $90 \mathrm{ml} \mathrm{F-12,} 100 \mu \mathrm{l} \mathrm{Mito}{ }^{\mathrm{TM}}+$ serum extender $(\mathrm{v} / \mathrm{v}, 1: 20), 5.0 \mathrm{ml} \mathrm{FBS}$, and $1.0 \mathrm{ml}$ PSN. F-12 medium contained $1000 \mathrm{ml}$ D-MEM/F-12, $1.2 \mathrm{~g} \mathrm{NaHCO}_{3}$, and $7.5 \mathrm{mmol} / \mathrm{L}$ HEPES with $\mathrm{pH}$ adjusted to 7.60 using $1.0 \mathrm{~mol} / \mathrm{L} \mathrm{NaOH}$.

\section{Preparation of isolated vagal ganglion neurons from neonatal rats}

To isolate neonatal VGNs, 4-7-day-old rat pups were used. Six neonatal pups of each gender were 
used for isolated cell preparations (2 pups for each preparation), and gender was verified surgically by either testis or ovary immediately after the bilateral ganglia were removed. All procedures (31) were identical to the isolation of adult VGNs except for the enzyme used (5 $\mathrm{mg} / \mathrm{ml}$ trypsin-3X; Worthington, Lakewood, NJ, USA) and incubation time (20 min).

\section{Neuron identification}

Because all patch-clamp recordings were performed on nodose neurons isolated from adult or neonatal rats, neuron/fiber type classification could not be determined by the afferent fiber conduction velocity. All tested neurons were identified as myelinated A- and Ah- and unmyelinated C-types based on validated standard AP parameters (Fig. 1), which were established based on data collected from slice preparation (32) combined with morphologic characteristics observed under a light microscope (33). Briefly, a combination of 4 parameters in our validated standard are useful for neuron classification, such as the action potential (AP) firing threshold, maximal upstroke velocity, AP duration measured at $50 \%$ height of amplitude $\left(\mathrm{APD}_{50}\right)$, and the repolarization "hump". In this experiment, neurons that showed a negative $\mathrm{AP}$ firing threshold $<-40 \mathrm{mV}$, maximal upstroke velocity $\left(\mathrm{UV}_{\mathrm{MAX}}\right)>250 \mathrm{mV} / \mathrm{ms}$, and $\mathrm{APD}_{50}$ $<1.0 \mathrm{~ms}$ without a repolarization "hump" were definitely classified as myelinated A-type VGNs (Fig. 1A $\&$ D). Neurons that showed a negative AP firing threshold $<-35 \mathrm{mV}, \mathrm{UV}_{\mathrm{MAX}}>250 \mathrm{mV} / \mathrm{ms}$ and $\mathrm{APD}_{50}$ $<2.0 \mathrm{~ms}$ with a dramatic repolarization "hump" were classified as myelinated Ah-type VGNs (Fig. 1B \& E). Neurons that showed a negative AP firing threshold $>$ $-35 \mathrm{mV}$ with a depolarization speed $<200 \mathrm{mV} / \mathrm{ms}$ and $\mathrm{APD}_{50}>2.0 \mathrm{~ms}$ with a significant "hump" over the time course of repolarization were classified as unmyelinated C-type VGNs (Fig. 1C \& F). Morphological parameters (33) may be necessary to more accurately and efficiently classify neurons prior to obtaining electrophysiological recordings.

Myelinated Ah-type VGNs are gender-specifically distributed primarily in adult female rats and are rarely observed in adult male rats (34). To compare histamine-induced responses for each classification between male and female rats, an extensive effort was made to locate Ah-type VGNs in adult males. Although more than 90 selected recordings from 11 male rats were investigated throughout the entire experiment, only 5 recordings fell into the Ah-type category (Table 1).
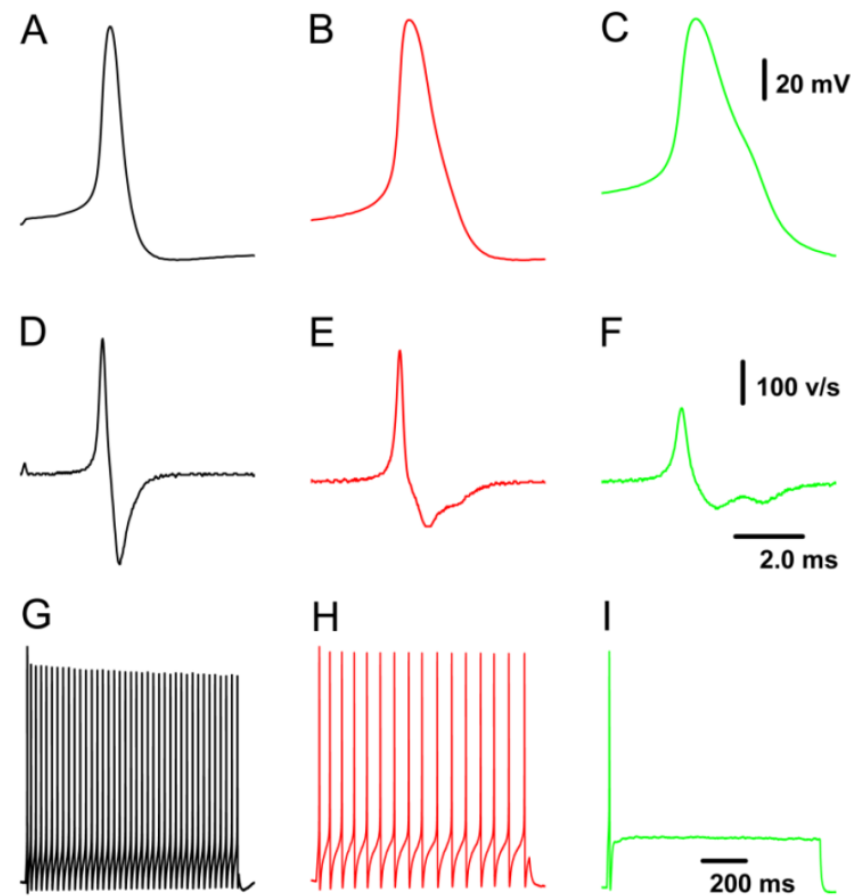

I

Figure I: Representative recordings of action potential (AP) and the pattern of repetitive discharge across populations of visceral ganglion neurons (VGNs) isolated from adult female rats. A - C: AP recordings elicited by a brief pulse from myelinated A-, Ah-, and unmyelinated $C$-type VGNs, respectively; $D$ - F: derivatives over the time course of APs shown in $(A-C)$. $G-I$ : Repetitive discharge evoked by the depolarization step ( $100 \mathrm{pA}$ for A-type, I50 pA for Ah-type, and $300 \mathrm{pA}$ for C-type) from the same VGN (shown in $A-C$ ). The vertical scale bars shown in $(C)$ and $(F)$ also applies to $(A),(B)$, and $(G)$ through $(I)$ and $(D) \&(E)$, respectively; the horizontal bar shown in $(F)$ also applies to $(A)$ through $(E)$.

\section{Electrophysiological techniques}

The whole-cell patch technique (35) was used for voltage- and current-clamp recording protocols using an Axoclamp 700A or 700B. Borosilicate glass pipettes (Sutter) were pulled and polished down to a resistance of 1.2-1.6 M $\Omega$. Following correction for all offsets, a giga-ohm seal was formed and the pipette capacitance was compensated. Total cell capacitance (30-50 pF) and electrode access resistance (3-5 M 2 ) were also compensated (60-80\%). For current-clamp recordings, a single AP was elicited using a $500-\mu \mathrm{s}$ current pulse delivered through the patch electrode, while step depolarizing currents were used to evoke repetitive discharge. After recording a single AP and repetitive discharge for neuron classification, the recording protocol was switched to either a current-clamp or voltage-clamp gap-free protocol to record histamine-induced membrane depolarization and inward currents. For histamine-induced membrane depolarization or inward current recordings, cells were maintained at a resting membrane potential near $-60 \mathrm{mV}$ or near zero currents by holding the voltage at $-60 \mathrm{mV}$. When membrane potentials or currents were 
stable in amplitude, a final concentration of $1.0 \mu \mathrm{M}$ histamine was applied to the cell through bath perfusion, and recordings were collected at least $1.0 \mathrm{sec}$ after histamine administration. Recordings were low pass filtered to $10 \mathrm{KHz}$ and digitized at $50 \mathrm{KHz}$. Experimental protocols, data collection, and preliminary analysis were performed using pCLAMP 10.3 and Digidata 1440A (Axon Instruments). Corrections for liquid junction potentials were taken into consideration before final data analysis.

\section{Recording solution}

$\mathrm{AP}$, membrane depolarization, and inward currents were investigated using the same extracellular solution composed of the following (in $\mathrm{mM}$ ): 137 $\mathrm{NaCl}, 5.4 \mathrm{KCl}, 1.0 \mathrm{MgCl}_{2}, 2.0 \mathrm{CaCl}_{2}, 10$ glucose, and 10 HEPES with $\mathrm{pH}$ adjusted to 7.35 using $1.0 \mathrm{~N} \mathrm{NaOH}$. The pipette solution for the recordings contained the following (in $\mathrm{mM}$ ): 140 potassium aspartate, 3.0 $\mathrm{MgCl}_{2}$, 4.0 BAPTA-K, 10 HEPES, and $0.25 \mathrm{CaCl}_{2}$ for a final buffered intracellular calcium concentration of $100 \mathrm{nM}$ with $\mathrm{pH}$ adjusted to 7.3 using $1.0 \mathrm{~N} \mathrm{KOH}$. Just before recording, $2.0 \mathrm{mM} \mathrm{Mg-ATP}$ was added to the pipette solution from a stock solution. Osmolarities of extracellular and pipette solutions were adjusted using D-mannitol (Sigma) to 310 and 290, respectively. A $100 \mu \mathrm{M}$ histamine stock solution was prepared fresh each day and applied using bath perfusion. All recordings were performed at room temperature $\left(20-23^{\circ} \mathrm{C}\right)$.

\section{Data acquisition and analysis}

In each data set, pulse-elicited single APs and step depolarization evoked repetitive discharge were sequentially recorded, and membrane depolarization or inward currents were observed in the same cell in the presence of histamine. Inward currents were normalized by whole-cell capacitance and described as the current density $(\mathrm{pA} / \mathrm{pF}$, Table 1$)$. For statistical analysis, specific types from each animal were averaged to yield one value per animal, which was entered as $n=1$ in the statistics package. Data from a minimum of 5 animals were included in this study. All averaged data for APs, firing frequency, and current density were expressed as the mean \pm SD, and statistical significance was evaluated using the $t$-test between groups. A two-tailed paired $t$-test was used to assess statistical significance $(P<0.05)$ of changes in current density before and after application of histamine. $P$ values less than 0.05 were considered significantly different.

\section{RESULTS}

AP parameters, such as AP firing threshold (APFT), maximal upstroke velocity ( $\left.\mathrm{UV}_{\mathrm{MAX}}\right), \mathrm{AP}$ duration $\left(\mathrm{APD}_{50}\right)$, and discharge character of $\mathrm{AP}$ firing frequency (APFF), are summarized in Table 1. These data show that AP parameters and discharge patterns were not significantly different between males and females (Fig. 1 and Table 1). However, AP duration $\left(\mathrm{APD}_{50}\right)$ was shorter $(P<0.01)$ with a faster downstroke velocity $\left(\mathrm{DV}_{\mathrm{APD} 50}, P<0.05\right)$ in OVX females than intact females (Table 1). Changes in other parameters were not confirmed. Although ovariectomy did not affect the behavior of myelinated A- and unmyelinated C-type VGNs, the neuroexcitability of myelinated Ah-type VGNs was dramatically decreased in OVX females. Importantly, we showed that myelinated Ah-type VGNs are a gender-specific subpopulation in females and are rarely observed in males. For comparison, 91 VGNs were tested from 11 adult male rats and only 5 were identified as myelinated Ah-types. The vast majority was myelinated A-type and fewer were unmyelinated C-type in males.

Table I: Discharge action potential parameters recorded from isolated adult male, female, and OVX female rats. Data are presented as the mean $\pm S D, n=$ the number of rats. ${ }^{*} P<0.05$ and ${ }^{* *} P<0.01$ vs adult female. $\dagger 5$ Ah-type neurons were observed in more than 90 selected recordings from II adult male rats.

\begin{tabular}{|c|c|c|c|c|c|c|c|c|c|}
\hline & \multicolumn{3}{|c|}{ Adult Male } & \multicolumn{3}{|c|}{ Adult Female } & \multicolumn{3}{|c|}{ Adult OVX Female } \\
\hline & A-type & Ah-type & C-type & A-type & Ah-type & C-type & A-type & Ah-type & C-type \\
\hline RMP & $-62.2 \pm 1.6$ & $-63.0 \pm 1.9$ & $-61.2 \pm 2.2$ & $-61.8 \pm 2.0$ & $-62.2 \pm 1.9$ & $-61.0 \pm 2.1$ & $-62.7 \pm 1.1$ & $-62.9 \pm 1.4$ & $-60.7 \pm 2.3$ \\
\hline APFT & $-43.3 \pm 3.1$ & $-41.5 \pm 2.3$ & $-28.8 \pm 3.3$ & $-41.9 \pm 2.7$ & $-43.4 \pm 2.2$ & $-28.6 \pm 2.3$ & $-42.0 \pm 2.4$ & $-43.4 \pm 1.2$ & $-27.8 \pm 2.4$ \\
\hline APFF & $38.6 \pm 3.4$ & $16.7 \pm 3.4$ & $2.4 \pm 1.4$ & $39.8 \pm 4.5$ & $18.5 \pm 3.6$ & $2.5 \pm 0.4$ & $37.3 \pm 5.7$ & $3.7 \pm 1.7^{* *}$ & $1.58 \pm 1.1$ \\
\hline $\mathrm{APD}_{50}$ & $0.81 \pm 0.14$ & $1.73 \pm 0.3$ & $2.63 \pm 0.4$ & $0.78 \pm 0.23$ & $1.67 \pm 0.3$ & $2.59 \pm 0.3$ & $0.78 \pm 0.3$ & $1.34 \pm 0.3^{* *}$ & $2.52 \pm 0.4$ \\
\hline AРPEAK & $53.1 \pm 3.0$ & $60.2 \pm 2.9$ & $60.7 \pm 2.7$ & $55.4 \pm 2.6$ & $62.1 \pm 2.7$ & $62.0 \pm 2.4$ & $53.7 \pm 4.0$ & $61.3 \pm 2.4$ & $62.4 \pm 2.0$ \\
\hline $\mathrm{AHP}_{80}$ & $16.2 \pm 2.6$ & $97.4 \pm 16$ & $108 \pm 19$ & $18.4 \pm 5.1$ & $51.4 \pm 11$ & $106 \pm 11$ & $22.1 \pm 3.6$ & $54.6 \pm 10$ & $114 \pm 17$ \\
\hline AHР & $-65.0 \pm 1.7$ & $-65.1 \pm 1.5$ & $-65.4 \pm 2.2$ & $-65.5 \pm 1.5$ & $-65.3 \pm 1.8$ & $-65.5 \pm 0.84$ & $-65.2 \pm 1.5$ & $-65.4 \pm 1.2$ & $-65.1 \pm 1.1$ \\
\hline UV $_{\text {APD50 }}$ & $214 \pm 18$ & $108 \pm 14$ & $54.3 \pm 15$ & $215 \pm 14$ & $113 \pm 14$ & $59.2 \pm 9.2$ & $210 \pm 12$ & $116 \pm 14$ & $53.7 \pm 7.7$ \\
\hline DV $_{\text {APD50 }}$ & $87.3 \pm 9.6$ & $-59.1 \pm 6.1$ & $-33.1 \pm 5.1$ & $115 \pm 18$ & $-61.4 \pm 4.2$ & $-35.7 \pm 3.2$ & $111 \pm 13$ & $73.8 \pm 3.1^{*}$ & $-37.0 \pm 5.2$ \\
\hline$n$ & 5 & $11 \dagger$ & 5 & 5 & 6 & 5 & 5 & 5 & 5 \\
\hline
\end{tabular}

Definitions: RMP: Resting membrane potential (mV). APFT: AP firing threshold (mV). APFF: AP firing frequency (Hz). APD 50 : AP duration at $50 \%$ deflection (msec). $\mathbf{A P}_{\text {Peak }}$ AP peak (mV). AHP Peak: Peak deflection of after-hyperpolarization (mV). AHP 80 : Time for $80 \%$ recovery to RMP after AHP (msec). $\mathbf{U V}_{\text {APD50: }}$ Upstroke velocity at $\mathrm{APD}_{50}(\mathrm{mV} / \mathrm{msec})$. DV $\mathrm{APD}_{50}$ : Downstroke velocity at $\mathrm{APD}_{50}(\mathrm{mV} / \mathrm{msec})$ 

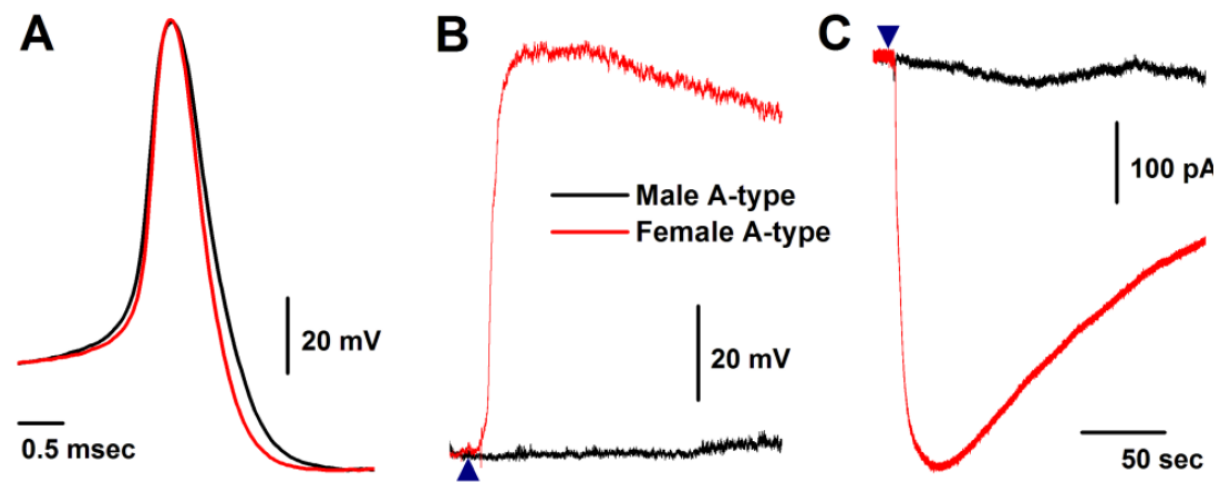

Figure 2: Histamine-induced membrane depolarization and inward currents in myelinated A-type vagal ganglion neurons (VGNs) isolated from adult male (black) and female (red) rats. A: representative action potentials (AP) elicited from A-types by a brief pulse; B: $1.0 \mu$ M histamine-induced membrane depolarization from resting membrane potential (RMP) near $-60 \mathrm{mV}$ observed on the same neurons shown in $(A)$ using the gap-free protocol under current-clamp mode; C: I.0 $\mu \mathrm{M}$ histamine-induced inward currents using the gap-free protocol with holding potential at $-60 \mathrm{mV}$ under voltage-clamp mode; $(\Delta)$ represents the starting points of bath-perfusion of histamine. Horizontal scale bar shown in (C) also applies to (B).

\section{Histamine-induced membrane depolarization and inward currents in myelinated A-type VGNs isolated from adult rats}

Seven myelinated A-type VGNs from males $(n=$ $5)$ and females rats $(n=5)$ were tested with $1.0 \mu \mathrm{M}$ histamine (Fig. 2A). None of the A-types from males were depolarized by histamine (Fig. 2B) and almost no inward currents were confirmed in the presence of histamine (Fig. 2C). This observation from male rats is consistent with reports in the literature (24). Surprisingly, all myelinated A-type VGNs from intact females tested responded to the same concentration of histamine under the same experimental conditions. Membrane depolarization (Fig. 2B) and inward currents (Fig. 2C) were both clearly observed upon administration of histamine. Average peak inward currents $(\mathrm{pA} / \mathrm{pF})$ in female A-types were dramatically larger $(8.74 \pm 3.29, P<0.01)$ than in males $(0.17 \pm 0.09)$, and nearly doubled $(18.8 \pm 3.47, P<0.01)$ in OVX females (Fig. 3A).

\section{Histamine-induced membrane depolarization and inward currents in myelinated Ah-type VGNs isolated from adult rats}

Only 5 VGNs out of 91 recordings in 11 males were confirmed as the Ah-type (Fig. 4A red and Table 1), while 9 Ah-type VGNs were observed (Fig. 4A black and Table 1$)$ from intact females $(n=6)$. Interestingly, all Ah-type VGNs from females and males were depolarized up to $\sim-30 \mathrm{mV}$ from the resting membrane potential (RMP, $\sim-60 \mathrm{mV}$ ), and a burst AP was observed in the initial phase of depolarization by histamine in some of the recordings (Fig. 4B). Immediately after running brief pulse and step depolariza- tion under current-clamp configuration, a gap-free voltage-clamp protocol was performed to record inward currents in the presence of histamine (Fig. 4C). Averaged data (Fig. 3A) showed that the peak inward currents $(\mathrm{pA} / \mathrm{pF})$ of the Ah-types were slightly larger in females $(9.96 \pm 3.66 \mathrm{pA} / \mathrm{pF}, n=6)$ compared with males $(4.25 \pm 2.17 \mathrm{pA} / \mathrm{pF}, n=5)$, but the difference was not significant $(P=0.056)$ due perhaps to the lower number of observations in males. In OVX females, the membrane potential was depolarized by histamine, whereas the histamine-induced peak inward current was significantly down-regulated $(2.75$ $\pm 1.16 \mathrm{pA} / \mathrm{pF}, n=5 P<0.05)$ compared with intact females (Fig. 3A).
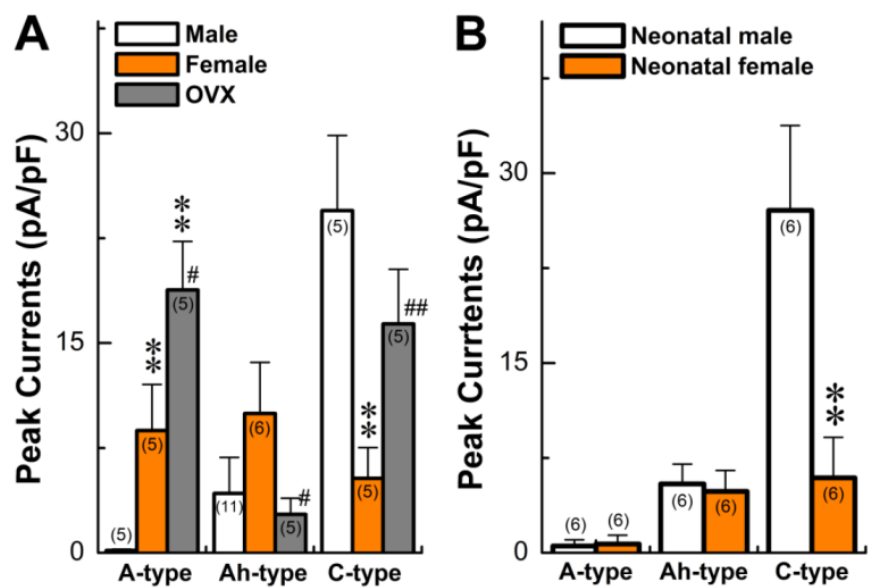

Figure 3: Summary data of histamine-induced inward currents from myelinated $\mathrm{A}$-, $\mathrm{Ah}$, and unmyelinated $\mathrm{C}$-type vagal ganglion neurons (VGNs) isolated from adult (A) and neonatal (B) rats. Current density was normalized by whole-cell capacitance and expressed as $\mathrm{pA} / \mathrm{pF}$. Average data are presented as the mean $\pm S D, * * P<0.01$ vs male, ${ }^{*} P<0.05$ and ${ }^{\#} P$ $<0.0$ I vs male. The number in the bracket shown in each column represents the total number of rats. 

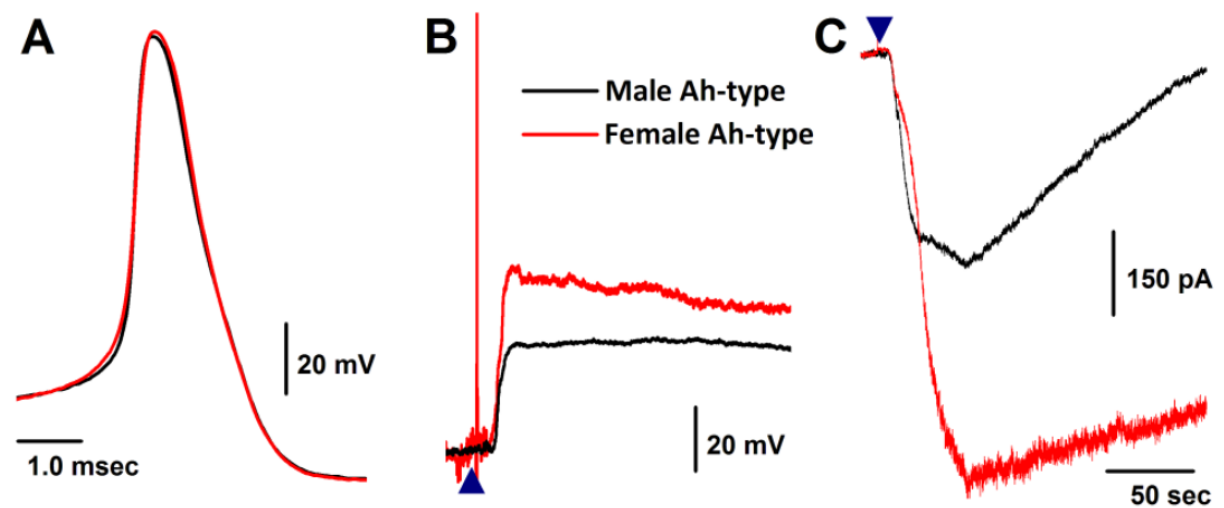

Figure 4: Histamine-induced membrane depolarization and inward currents in myelinated Ah-type vagal ganglion neurons (VGNs) isolated from adult male (black) and female (red) rats. A: representative action potentials (AP) elicited from Ah-type APs by a brief pulse; B: I.0 $\mu$ M histamine-induced membrane depolarization from resting membrane potential (RMP) near $-60 \mathrm{mV}$ observed on the same neurons shown in $(A)$ using the gap-free protocol under current-clamp mode; C: I.0 $\mu \mathrm{M}$ histamine-induced inward currents using the gap-free protocol with holding potential at $-60 \mathrm{mV}$ under voltage-clamp mode; $(\Delta)$ represents the starting points of bath-perfusion of histamine. The horizontal scale bar shown in (C) also applies to (B).

\section{Histamine-induced membrane depolarization and inward current in unmyelinated C-type VGNs isolated from adult rats}

Similar to myelinated Ah-type VGNs, all unmyelinated C-type VGNs identified from males (Fig. 5A), intact females (Fig. 5A), and OVX females (data not shown) responded to $1.0 \mu \mathrm{M}$ histamine, manifested as membrane depolarization (Fig. 5B). Depolarization induced by histamine was almost twice as large in males (up to $\sim-20 \mathrm{mV}$ ) than intact females (up to $\sim-40$ $\mathrm{mV}$ ). This was equivalent to the effect of histamine on inward currents (Fig. 5C), manifested as significant peak inward current in males $(>25 \mathrm{pA} / \mathrm{pF}$ average, Fig. 3A). The peak inward current in females was 5 times $(\sim 5 \mathrm{pA} / \mathrm{pF}$ average) lower compared with that observed in males $(P<0.01$, Fig. 3A). In OVX females, histamine-induced peak inward currents were partially restored $(\sim 20 \mathrm{pA} / \mathrm{pF})$ compared with males $(P>$ $0.05)$ and dramatically increased compared with intact females $(P<0.01$, Fig. 3A).

\section{Changes in histamine-induced responses of myelinated and unmyelinated VGNs isolated from OVX female rats}

In OVX females, AP parameters and discharge patterns in myelinated A- and unmyelinated C-type VGNs were not altered, although the $\mathrm{APD}_{50}$ was reduced $(P<0.01)$ and DV APD50 was increased $(P<0.05)$ compared with intact females (Table 1). There was a significant reduction in discharge frequency of Ah-type VGNs from $\sim 20 \mathrm{~Hz}$ in intact females down to less than $5.0 \mathrm{~Hz}$ in OVX females $(P<0.01$, Table 1$)$. These data are consistent with our previous observations $(34,36,37)$. In addition, $1.0 \mu \mathrm{M}$ histamine induced membrane depolarization of myelinated A-, Ah-, and unmyelinated C-types (data not shown). Histamine-induced peak inward currents observed across all VGN populations were markedly altered in OVX females compared with intact females. Averaged peak inward currents $(\mathrm{pA} / \mathrm{pF})$ increased overall (Fig. $3 \mathrm{~A})$ in both myelinated A- $(P<0.05)$ and unmyelinated C-type VGNs $(P<0.01)$, while the peak inward currents of myelinated Ah-types in OVX females were only about one-fourth the average current density $(2.75 \pm 1.16 \mathrm{pA} / \mathrm{pF}, \mathrm{P}<0.05)$ compared with intact females $(9.96 \pm 3.66 \mathrm{pA} / \mathrm{pF})(P<0.05$, Fig. $3 \mathrm{~A})$.

\section{OVX alters histamine sensitivity across all VGN populations isolated from adult intact and OVX female rats}

As shown, $1.0 \mu \mathrm{M}$ histamine induced membrane depolarization from the resting membrane potential across all VGN populations in intact females. Because a determination as to which type of VGN is more sensitive to histamine cannot be made based on this observation, a lower concentration of histamine (300 $\mathrm{nM}$ ) was applied to another set of VGNs (Fig. 6) isolated from intact and OVX females. Membrane depolarization induced by $300 \mathrm{nM}$ histamine was only confirmed in myelinated Ah-type VGNs (Fig. 6B), with an average depolarization of $14.5 \pm 3.24 \mathrm{mV}(n=$ 5). Similar responses were not observed in either myelinated A- $(n=5)$ or unmyelinated C-type VGNs $(n=$ 5) (Fig. 6A \& C). A similar test was performed on all VGN types isolated from OVX females. Membrane potentials in both myelinated A- $(n=5$ OVX; Fig. $6 \mathrm{~A}$, red) and unmyelinated C-type VGNs ( $n=6$ OVX; Fig. 6C) were significantly depolarized by $300 \mathrm{nM}$ histamine up to $\sim-40 \mathrm{mV}$ from resting stages $(P<0.01)$. The membrane potential in myelinated Ah-types was only slightly depolarized $(-58.2 \pm 2.3 \mathrm{mV}$ with histamine, $n$ $=5$ OVX vs. $-62.6 \pm 1.6 \mathrm{mV}$ before histamine, $P>0.05$ ) under the same experimental conditions (Fig. 6B) and was dramatically lower in magnitude compared with intact females (Fig. 6B). 

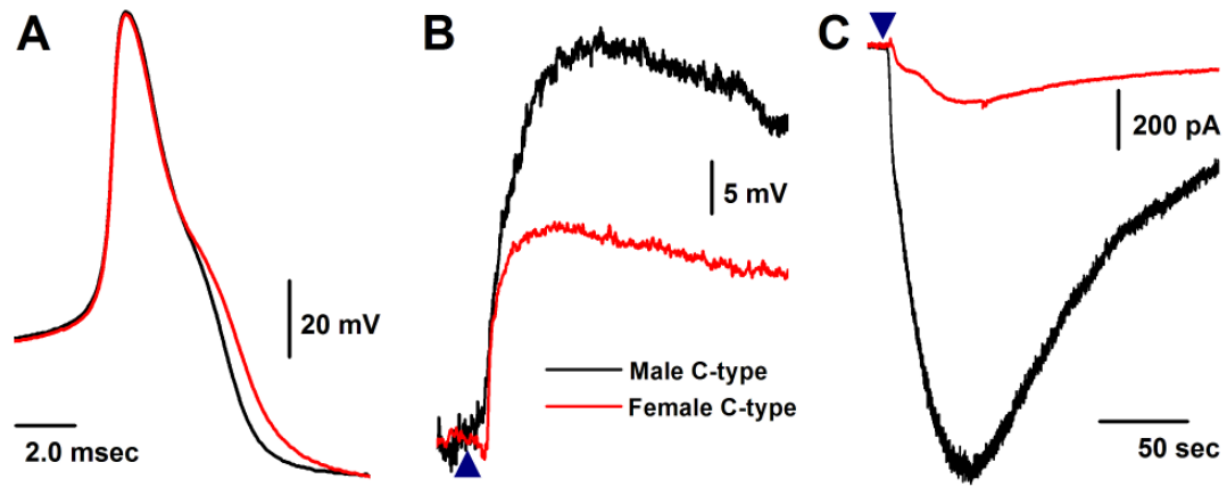

Figure 5: Histamine-induced membrane depolarization and inward currents in unmyelinated C-type vagal ganglion neurons (VGNs) isolated from adult male (black) and female (red) rats. A: representative action potentials (AP) elicited from C-type VGNs by a brief pulse; B: I.0 $\mu$ M histamine-induced membrane depolarization from resting membrane potential (RMP) near $-60 \mathrm{mV}$ observed on the same neurons shown in (A) using the gap-free protocol under current-clamp mode; C: I.0 $\mu \mathrm{M}$ histamine-induced inward currents using the gap-free protocol with holding potential at $-60 \mathrm{mV}$ under voltage-clamp mode; $(\mathbf{A})$ represents the time points of bath-perfusion of histamine. The horizontal scale bar shown in (C) also applies to (B).
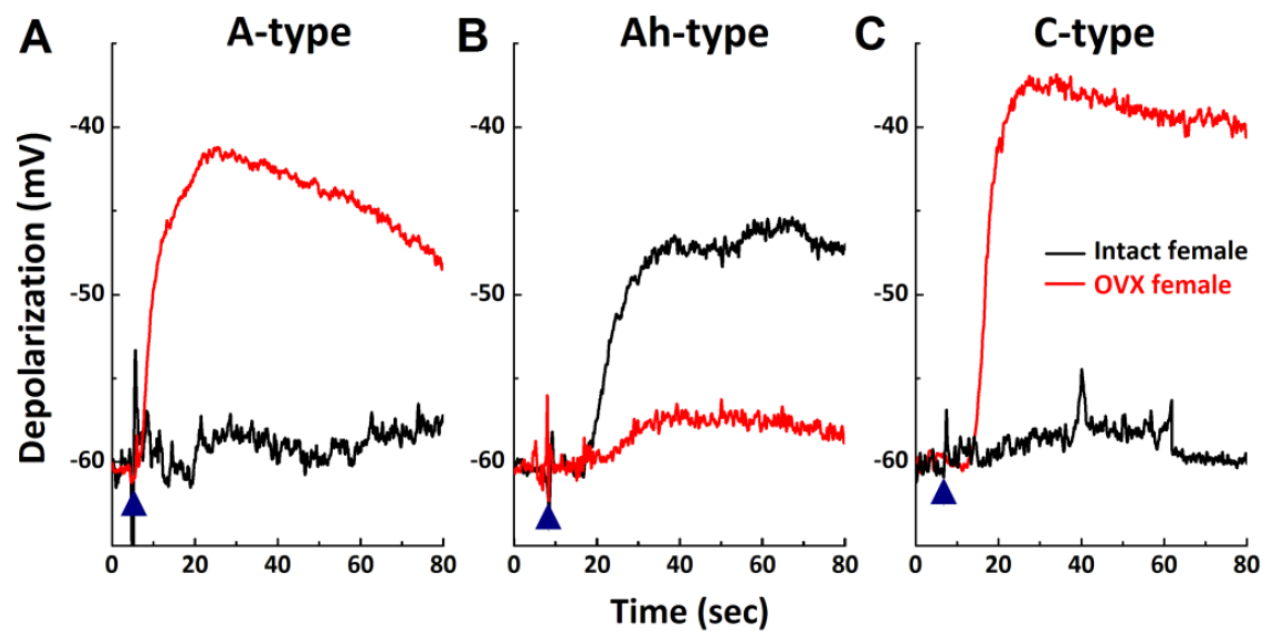

Figure 6: High sensitivity of Ah-type vagal ganglion neurons (VGNs) to histamine and down-regulation in ovariectomized (OVX) female rats. Myelinated A-, Ah-, and unmyelinated C-type VGNs were first identified and membrane depolarization was observed in the presence of $300 \mathrm{nM}$ histamine using the gap-free protocol under current-clamp mode on the same neuron. A - C: represents $300 \mathrm{nM}$ histamine-induced membrane depolarization in A-, Ah-, and C-type VGNs, respectively, from intact (black) or OVX female (red) rats; $(\boldsymbol{\Delta})$ represents the starting points of bath-perfusion of histamine.

\section{Developmental changes and gender differ- ences in histamine-induced inward currents in VGNs isolated from neonatal rats}

The prevalence of asthma has been shown to be higher in younger boys than girls, with a reversal pattern after adolescence. This led to questions regarding differences in the chemosensitivity of VGNs to histamine in neonatals compared to adults. Recent findings have shown that myelinated Ah-types are equally distributed in both neonatal male and female rats $(32,34)$. Comparing histamine-induced inward currents of VGNs between neonatal male and female rats and data observed in adults was performed. Results (Fig. 3B) indicated that very small but detectable depolarizations (data not shown) were observed in A-types from both sexes with no difference $(P>0.05)$ in current density. Histamine definitely induced larger inward currents of Ah-types in either male or female rats with identical amplitudes $(P>0.05)$.
However, significant larger inward currents $(29.1 \pm$ $7.71 \mathrm{pA} / \mathrm{pF}, n=6$ ) were confirmed in male C-types, which were 5 times larger than in female C-types (5.63 $\pm 4.2 \mathrm{pA} / \mathrm{pF}, n=6, P<0.01)$. The patterns of histamine-induced inward currents in all neonatal VGN populations were quite different from adults (Fig. 3A).

\section{Discussion}

\section{Gender differences in chemosensitivity of va- gal ganglion neurons to histamine in adult rats}

Using VGNs isolated from adult rats and an established rat model of experimentally induced postmenopausal estrogen deficiency (38) combined with electrophysiological and pharmacological approaches, we show for the first time significant gender differences in the chemosensitivity of VGNs to histamine. With regard to adult males, our observations are consistent with results in the literature (24) in 
which histamine induced membrane depolarization in all of the unmyelinated C-type VGNs tested and none of the myelinated A-type VGNs. In contrast in adult female rats, not only unmyelinated C-types, but also myelinated A-types and gender-specific subpopulations of myelinated Ah-type VGNs $(32,34)$ responded to histamine, as suggested by significant membrane depolarization and marked inward currents in the presence of histamine. These major findings may explain, at least in part, that histamine could be a key player in gender-based differences in autonomic reflex function under physiological and disease conditions $(5,6)$. Consistent with our previous observation (34), a very low percentage of likely myelinated Ah-type neurons from male rats were verified using standard validation (32) and morphological parameters (33) in this investigation. However, male Ah-types do not follow the same pharmacological tendency (34) as female Ah-types regarding positive reactivity to a $\beta$-methyl-adenosine triphosphate and negative reactivity to capsaicin. Although male Ah-types showed a similar but less potent response to histamine, the functional roles of this class of neurons may not be significant.

\section{Gender differences in chemosensitivity of va- gal ganglion neurons to histamine in neonatal rats}

Clinical evidence has shown that asthma is more common in boys than in girls, although the prevalence switches in adults or after adolescence $(29,30,39-42)$. This pattern raised questions about changes in the chemosensitivity of VGNs to histamine over time. In this regard, sensitivity to histamine was tested using VGNs isolated from male and female neonatal rats. Compared with adult rats, myelinated A-, Ah-, and unmyelinated C-type VGNs were equally distributed in both neonatal males and females (32). Using this model, we can easily compare and evaluate chemosensitivity to histamine across all neuronal populations and predict the impacts of different neurons with histamine function during the early stages of life. On average, histamine-induced inward currents in Aand Ah-types from neonatal males were equivalent with those observed in neonatal females, while the current density of C-types induced by histamine was at least 5 times larger in neonatal males than neonatal females. This suggests that the patterns of histamine-induced events are dramatically different compared with data from adult rats and that C-type VGNs may play a dominant role, especially in neonatal male rats. This observation, in combination with current data from adult animals, strongly supports the notion that the prevalence of asthma is higher in boys than girls (43) in childhood and may partially explain the reversal pattern of asthma prevalence during the lifespan.

\section{Coordinated changes in chemosensitivity to histamine in neuronal populations in females following ovariectomy}

Menopausal transition is a critical time period during the lifespan of a woman. Gender differences in histamine sensitivity involved in pathophysiological and disease conditions are well recognized, and as such, changes during the menopausal transition period are expected. To fully understand the plausible mechanisms underlying this transition, the rat model in which bilateral ovaries are removed was selected to simulate the transition to menopause. Unlike male rats, histamine induced equivalent responses in all types of VGNs from intact females, as shown by current density measurements ( $P>0.05$ by ANOVA). These data are the first direct evidence from female rats indicating that myelinated and unmyelinated VGNs may contribute equally to histamine-related functions, while C-types are the only histamine-related neurons that play a role in males, suggesting that this may be a major factor leading to gender differences in histamine-related functions and diseases. Interestingly, the present data demonstrate that the sensitivity of female VGNs to histamine changed following an ovariectomy, manifested as a significant decrease in histamine-induced current density in Ah-types (Fig. 3A), but increase in C-types with a marked reduction in neuroexcitability shown by repetitive discharge profiles (36). The fashion of current density of Ah- vs C-types in OVX female rats became more like those observed in A- vs C-types in males, implying that histamine-related physiological functions change in female rats following OVX. Involvement of histamine as a nonadrenergic and noncholinergic factor (44) at the level of neurocontrol of circulation may partially explain why blood pressure in postmenopausal women becomes identical to age-matched men $(6,45)$. In this study, A- and Ah-type VGNs are both myelinated neurons; however, histamine-induced current density was enhanced in A-types, rather than reduced as in Ah-types by OVX. This observation may be explained as compensation for down-regulation of histamine-related activity in Ah-types after OVX.

To further verify changes in sensitivity to histamine across populations of VGNs from female rats, lower concentrations of histamine were applied. Under the same experimental conditions, membrane depolarization was only confirmed in Ah-type VGNs without significant changes in both A- and C-types. After surgically and bilaterally removing the ovaries, histamine-induced membrane depolarization 
dropped to near resting $(-60 \mathrm{mV})$ instead of increasing in both A- and C-types. These data further indicate that myelinated Ah-type VGNs are more sensitive to histamine and may play major roles in female reflex functions and that this activity may be lost due to lack of female hormone support.

\section{Future study}

Histamine receptor subtypes in each neuron category are currently under investigation using pharmacological testing with selective agonists or antagonists for histamine. Molecular approaches, such as single-cell RT-PCR, to evaluate messenger RNA expression in single neurons is another possible experiment to avoid contamination from other neuron types in the ganglion tissue. Pharmacological and molecular investigations together with immunohistochemical analysis using specific antibodies would be used to further confirm these conclusions.

\section{Conclusion}

This study provides the first evidence demonstrating gender differences in chemosensitivity of vagal ganglion neurons to histamine, which may be a major contributor to gender differences in reflex functions, cardiovascular regulation, asthma, and neuropathic nociception.

\section{Acknowledgements}

This project was supported by research grants from the Natural Science Foundation of China (No.: 30973532; 81173051; 31171122) to G-F Qiao and B-Y Li and the Education Department of China (No.: 20112307110008) to B-Y Li.

\section{Competing Interests}

The authors have declared that no competing interest exists.

\section{References}

1. Frei R, Ferstl R, Konieczna P, Ziegler M, Simon T, Rugeles TM, et al. Histamine receptor 2 modifies dendritic cell responses to microbial ligands. J Allergy Clin Immunol. 2013; 132: 194-204

2. Thomas CM, Hong T, van Pijkeren JP, Hemarajata P, Trinh DV, Hu W, et al. Histamine derived from probiotic Lactobacillus reuteri suppresses TNF via modulation of PKA and ERK signaling. PloS One. 2012;7(2): e31951.

3. Palomero-Gallagher N, Zilles K. Neurotransmitter receptor alterations in hepatic encephalopathy: A review. Arch Biochem Biophys. 2013; 536(2): 109-21.

4. McCoy ES, Taylor-Blake B, Zylka MJ. CGRPalpha-expressing sensory neurons respond to stimuli that evoke sensations of pain and itch. PloS One. 2012; 7(5): e36355.

5. Quagliotto E, Neckel H, Riveiro DF, Casali KR, Mostarda C, Irigoyen MC, et al. Histamine in the posterodorsal medial amygdala modulates cardiovascular reflex responses in awake rats. Neuroscience. 2008; 157(4): 709-19.

6. Bhuiyan ME, Waki H, Gouraud SS, Takagishi M, Kohsaka A, Maeda M. Histamine receptor $\mathrm{H} 1$ in the nucleus tractus solitarii regulates arterial pressure and heart rate in rats. Am J Physiol Heart Circ Physiol. 2011; 301(2): H523-9.

7. Coppola G, Caccamo G, Bacarella D, Corrado E, Caruso M, Cannavo MG, et al. Vasospastic angina and scombroid syndrome: a case report. Acta Clin Belg. 2012; 67(3): 222-5.
8. Hosokawa S, Hiasa Y, Murakami N, Tobbeto Y, Nakagawa T, Chen P, et al. The impact of gender difference on the effects of preinfarction angina on microvascular damage with reperfused myocardial infarction. Clin Cardiol. 2010; 33(7): 412-7.

9. Yu J, Lou GD, Yue JX, Tang YY, Hou WW, Shou WT, et al. Effects of histamine on spontaneous neuropathic pain induced by peripheral axotomy. Neurosci Bull. 2013; 29(3): 261-9.

10. Rosa AC, Fantozzi R. The role of histamine in the neurogenic inflammation. Br J Pharmacol. 2013; 170(1): 38-45.

11. Stewart WF, Lipton RB, Celentano DD, Reed ML. Prevalence of migraine headache in the United States. Relation to age, income, race, and other sociodemographic factors. JAMA. 1992; 267(1): 64-9.

12. Stewart WF, Wood C, Reed ML, Roy J, Lipton RB. Cumulative lifetime migraine incidence in women and men. Cephalalgia. 2008; 28(11): 1170-8.

13. Luo T, Chen B, Zhao Z, He N, Zeng Z, Wu B, et al. Histamine H2 receptor activation exacerbates myocardial ischemia/reperfusion injury by disturbing mitochondrial and endothelial function. Basic Res Cardiol. 2013; 108(3): 342.

14. Zdravkovic V, Pantovic S, Rosic G, Tomic-Lucic A, Zdravkovic N, Colic M, et al. Histamine blood concentration in ischemic heart disease patients. J Biome Biotechnol. 2011; 2011: 315709.

15. Rao SP, Dunbar JC. A role for the central histaminergic system in the leptin-mediated increase in cardiovascular dynamics. Brain Res Bull. 2005; 64(5): 425-32.

16. Halliwill JR, Buck TM, Lacewell AN, Romero SA. Postexercise hypotension and sustained postexercise vasodilatation: what happens after we exercise? Exp Physiol. 2013; 98(1): 7-18

17. Salcedo $C$, Pontes $C$, Merlos M. Is the $\mathrm{H} 4$ receptor a new drug target for allergies and asthma? Front Biosci (Elite Ed). 2013; 5: 178-87.

18. Jones BL, Kearns G, Neville KA, Sherwin CM, Spigarelli MM, Leeder JS. Variability of Histamine Pharmacodynamic Response in Children With Allergic Rhinitis. J Clin Pharmacol. 2013; 53(7): 731-7.

19. Nugent L, Mehta PK, Bairey Merz CN. Gender and microvascular angina. J Thromb Thrombolysis. 2011; 31(1): 37-46.

20. Leynaert B, Sunyer J, Garcia-Esteban R, Svanes C, Jarvis D, Cerveri I, et al. Gender differences in prevalence, diagnosis and incidence of allergic and non-allergic asthma: a population-based cohort. Thorax. 2012; 67(7): 625-31.

21. Choi IS. Gender-specific asthma treatment. Allergy Asthma Immunol Res. 2011; 3(2): 74-80

22. Pethica BD, Tomlin A, Hall J, Penrose A, Frost G. Sex difference in prescription of asthma drugs is smaller than previously found. BMJ. 1999; 319(7206): 385.

23. Chen W, Mempel M, Schober W, Behrendt H, Ring J. Gender difference, sex hormones, and immediate type hypersensitivity reactions. Allergy. 2008; 63(11): 1418-27.

24. Higashi $\mathrm{H}$, Ueda $\mathrm{N}$, Nishi $\mathrm{S}$, Gallagher JP, Shinnick-Gallagher $\mathrm{P}$. Chemoreceptors for serotonin (5-HT), acetylcholine (ACh), bradykinin (BK), histamine $(\mathrm{H})$ and gamma-aminobutyric acid (GABA) on rabbit visceral afferent neurons. Brain Res Bull. 1982; 8(1): 23-32.

25. Yu S, Kollarik M, Ouyang A, Myers AC, Undem BJ. Mast cell-mediated long-lasting increases in excitability of vagal $\mathrm{C}$ fibers in guinea pig esophagus. Am J Physiol Gastrointest Liver Physiol. 2007; 293(4): G850-6.

26. Poole SL, Lewis DI, Deuchars SA. Histamine depolarizes neurons in the dorsal vagal complex. Neurosci Lett. 2008; 432(1): 19-24.

27. Thompson GW, Horackova M, Armour JA. Chemotransduction properties of nodose ganglion cardiac afferent neurons in guinea pigs. Am J Physiol Regul Integr Comp Physiol. 2000; 279(2): R433-9.

28. Jafri MS, Moore KA, Taylor GE, Weinreich D. Histamine H1 receptor activation blocks two classes of potassium current, IK(rest) and IAHP, to excite ferret vagal afferents. J Physiol. 1997; 503 ( Pt 3): 533-46.

29. Almqvist $C$, Worm M, Leynaert B. Impact of gender on asthma in childhood and adolescence: a GA2LEN review. Allergy. 2008; 63(1): 47-57.

30. Vink NM, Postma DS, Schouten JP, Rosmalen JG, Boezen HM. Gender differences in asthma development and remission during transition through puberty: the TRacking Adolescents' Individual Lives Survey (TRAILS) study. J allergy Clin immunol. 2010; 126(3): 498-504.

31. Li BY, Schild JH. Comparisons of somatic action potentials from dispersed and intact rat nodose ganglia using patch-clamp technique. Acta Pharmacol Sin. 2002; 23(6): 481-9.

32. Li BY, Schild JH. Electrophysiological and pharmacological validation of vagal afferent fiber type of neurons enzymatically isolated from rat nodose ganglia. J Neurosci Methods. 2007; 164(1): 75-85.

33. Lu XL, Xu WX, Yan ZY, Qian Z, Xu B, Liu Y, et al. Subtype identification in acutely dissociated rat nodose ganglion neurons based on morphologic parameters. Int J Bio Sci. 2013; 9(7): 716-27.

34. Li BY, Qiao GF, Feng B, Zhao RB, Lu YJ, Schild JH. Electrophysiological and neuroanatomical evidence of sexual dimorphism in aortic baroreceptor and vagal afferents in rat. Am J Physiol Regul Integr Comp Physiol. 2008; 295(4): R1301-10.

35. Li BY, Schild JH. Patch clamp electrophysiology in nodose ganglia of adult rat. J neurosci methods. 2002; 115(2): 157-67.

36. Qiao GF, Li BY, Lu YJ, Fu YL, Schild JH. 17 $\beta$-estradiol restores excitability of a sexually dimorphic subset of myelinated vagal afferents in ovariectomized rats. Am J Physiol Cell Physiol. 2009; 297(3): C654-64.

37. Qiao GF, Qian Z, Sun HL, Xu WX, Yan ZY, Liu Y, et al. Remodeling of hyperpolarization-activated current, ih, in ah-type visceral ganglion neurons following ovariectomy in adult rats. PloS One. 2013;8(8):e71184 
38. Tabarean IV, Sanchez-Alavez M, Sethi J. Mechanism of $\mathrm{H}(2)$ histamine receptor dependent modulation of body temperature and neuronal activity in the medial preoptic nucleus. Neuropharmacology. 2012; 63(2): 171-80.

39. Eagan TM, Brogger JC, Eide GE, Bakke PS. The incidence of adult asthma: a review. Int J Tuberc lung Dis. 2005; 9(6): 603-12.

40. Becklake MR, Kauffmann F. Gender differences in airway behaviour over the human life span. Thorax. 1999; 54(12): 1119-38.

41. Melgert BN, Ray A, Hylkema MN, Timens W, Postma DS. Are there reasons why adult asthma is more common in females? Curr Allergy Asthma Rep. 2007; 7(2): 143-50.

42. Dodge RR, Burrows B. The prevalence and incidence of asthma and asthma-like symptoms in a general population sample. Am Rev Respir Dis. 1980; 122(4): 567-75.

43. Shamssain $M$. Trends in the prevalence and severity of asthma, rhinitis and atopic eczema in 6- to 7- and 13- to 14-yr-old children from the north-east of England. Pediatr Allergy Immunol. 2007; 18(2): 149-53.

44. Enok S, Simonsen LS, Pedersen SV, Wang T, Skovgaard N. Humoral regulation of heart rate during digestion in pythons (Python molurus and Python regius). Am J Physiol Regul Integr Comp Physiol. 2012; 302(10): R1176-83.

45. Crossley DA, 2nd, Sartori MR, Abe AS, Taylor EW. A role for histamine in cardiovascular regulation in late stage embryos of the red-footed tortoise, Chelonoidis carbonaria Spix, 1824. J Comp Physiol B. 2013; 183(6): 811-20. 